This is a manuscript submitted for publication in a journal and undergoing peer review

\title{
Trinity Review: Integrating Registered Reports with research ethics and funding reviews
}

Yuki Mori ${ }^{\dagger}$ (Kyushu University, aiyou403@gmail.com)

Kaito Takashima ${ }^{\dagger}$ (Kyushu University, ktaaa331@gmail.com)

Kohei Ueda (Kyushu University, ueda3156@gmail.com)

Kyoshiro Sasaki (Kansai University, kyoshiro0920@gmail.com)

Yuki Yamada (Kyushu University, yamadayuk@gmail.com)

\footnotetext{
$\dagger$ Equally contributed authors

$\ddagger$ Corresponding author
} 


\begin{abstract}
One major source of high exhaustion for researchers is the redundant paperwork of three different documents (research papers, applications for ethics review, and applications for research grants) for the same research plan. These similar documents are submitted to three different organizations, each with its own format, and independently peer-reviewed three times. This is a wasteful and redundant process for researchers. Here, we propose a trinity review that integrates scientific, ethics, and research funding reviews. In our proposed trinity review system, scientific and ethics reviews are undertaken concurrently for a research protocol before running the study. When the protocol is accepted in principle through these review processes, a funding review will occur, and researchers will conduct their studies. After experiments or surveys, the scientific review will be conducted for a completed version of the paper including results and discussions (i.e., the full paper) again, and the full paper will be published after it has passed the second review. This paper provides a detailed explanation of the operation of the trinity review system, discusses its positive impacts, and provides solutions to difficulties in its implementation. Moreover, we highlight the advantages of this system regarding reproducibility and research integrity.
\end{abstract}

Keywords: Registered Reports, Research ethics, Research grants, Peer review, Review system, Academic publishing. 


\section{Introduction}

Researchers are required to write three different documents for each project. One is a research paper manuscript. Research papers are academic publications that describe some of the achievements of a research project, and many researchers are most focused on producing these peer-reviewed publications. Recently, it has become relatively common to submit papers in the form of Registered Reports (RRs) [1,2]. RRs are an editorial system in which the authors submit a research protocol to a journal before running the study (Stage 1). During Stage 1, the value of the research question, the rationale for the hypothesis, and the validity of the methods are assessed [1]. This allows reviewers to assist authors in improving the protocol and rationale, or to make changes that will further improve the quality of the paper [3]. As a result of peer review and revision, if the protocol is judged to be publishable, the protocol and results are accepted in principle for publication, regardless of the results of studies performed afterward. After the studies, a completed version of the paper is submitted again for peer review, and a final decision on publication is made (Stage 2). This aspect of peer-reviewing the work before studies are conducted is distinctive and is the focus of this commentary, which will be discussed later.

The second piece of writing is an application for ethics review. In experimental psychology, for example, the field in which the authors specialize, researchers perform experiments on living things, including humans (i.e., subjects). To avoid violating the subjects' rights and safety, the study plan is reviewed by the ethical committee of affiliated institutions in advance. This is also true of any research involving humans or animals as subjects, such as medical research [4]. If the plan does not present ethical problems and is approved, researchers can start their studies [5]. The application for ethics review, similar to RRs, is peer-reviewed and often revised before studies are conducted.

Authors also often write applications for research grants to conduct their studies. Funds are necessary for research activities. To obtain them, researchers submit dozens of pages on the plan for their studies to funding agencies. If the agencies judge the plans as valuable and adopt them, the researchers obtain grants. Usually, researchers submit multiple applications to several agencies, as the failure of all applications could threaten the very existence of their laboratory. In many cases, different forms and contents must be prepared for each resubmission. A study reported that each grant proposal costs researchers an average of 34 working days [6]. The important thing here is that the grant application is also peer-reviewed and selected before the studies. 
As mentioned above, researchers submit similar documents to three different organizations, each with its own format, and they are independently peer-reviewed three times. This is a wasteful and redundant process. The research ethics and funding reviews also assess scientific validity and importance, which overlap with the peer review of academic papers, wasting the effort of the reviewers involved in all three. Hence, we propose to integrate RRs with research ethics and funding reviews. A system that integrates RRs with funding reviews has already been proposed [7, 8]. However, the proposed method significantly reduces researchers' workloads, which are caused by redundant writing tasks, more than the conventional system by integrating a research ethics review. This also completely separates the roles of reviewers engaged in ethical assessment and reviewers engaged in checking scientific validity and importance, which will result in a general reduction in their workload.

\section{Main text}

As mentioned above, this commentary proposes a trinity review that integrates Registered Reports, research ethics reviews, and research funding reviews. In the following, we describe the specific methods, the role of journals and communities, involvement of ethics reviewers, matching with agencies, and advantages and disadvantages.

Researchers submit a detailed pre-research protocol in the Stage 1 manuscript of the RRs. Generally, the protocol is peer-reviewed by several reviewers; this involves assessing the value of the research question, the hypothesis rationale, and the validity of the methods for testing the hypothesis. Note that for exploratory research RRs, it is also important to assess the validity of explorations and the transparency of its process after completing Stage 1 [9]. In our proposed trinity review system, an ethics review will be conducted concurrently for the Stage 1 protocol. Some reviewers dedicated to ethical aspects will be assigned to the protocol and review the ethical aspects of the paper. Protocols which have ethical problems are sent back to the authors and they have to revise them according to the reviewer's comments and the editor's decision. If there is some necessity in terms of ethical considerations that are not covered in the code, the protocol is reviewed additionally by the author's institution (e.g., in the medical field, institutional acknowledgement might be necessary if the research uses some invasive methods against humans). When the protocol is accepted through these review processes, a funding review would be initiated. Here, the Stage 1 protocols and funders would be matched in some way. The funders assess whether the Stage 1 protocols are possibly beneficial to them or the public and invest grants for protocols that meet their criteria. In this 
way, the trinity review system unifies the three different types of peer reviews and makes them run smoothly (Figure 1).

-- Figure 1 around here--

Who will manage the trinity review system? Considering that at its core is the RR, RRfriendly journals will be able to manage each protocol. Particularly, conducting ethics reviews at the same time as peer reviews can be implemented immediately. Otherwise, some peer review communities could participate in this endeavor. For example, Peer Community in (PCI) [10] could be one of the candidates. PCI is a scientific organization that offers an editorial process for preprints. In this process, researchers upload their preprint to a server (e.g., arXiv, bioRxiv, and PsyArXiv) and submit it to the corresponding thematic PCI. Then, a recommender (similar to an action editor in academic journals) is assigned to the preprint, and a process similar to the typical editorial and review processes begins. After the preprint is accepted through peer review, a short recommendation, reviews, and editorial correspondence are published on the corresponding thematic PCI. The manuscript accepted in the corresponding thematic PCI will be processed smoothly by journals (particularly, the Peer Community Journal and PCI-friendly journals). Recently, PCI has started handling RRs (i.e., PCI Registered Reports [11]); furthermore, PCI Registered Reports, as well as RRs-friendly journals, might work well with the trinity review system. As mentioned above, a trinity review system can be managed by the existing systems.

Funding reviewing (or matching) is probably the trickiest part of this system. The funding review would not require any revisions to the manuscript, and thus it only takes place after the protocol has passed scientific and ethics reviews and has been accepted in principle. Research funds are provided for protocols, not for individual researchers as in a typical funding review, and two ways of matching protocols and funders are possible. First, funders offer grants, these offerings are listed, and researchers apply for those that match their protocols. This is similar to the traditional grant system; however, it is qualitatively different, since the reviewed manuscripts guarantee the importance of the research question, relevance of the protocol, and research ethics. A second way is for funders to select and invest in the protocol they are interested in from a list of in-principle accepted protocols that can be browsed only by the funders. These methods help researchers to avoid spending time in wasting paper work, and funders can easily assess whether the research is truly suitable for funding. Note that this funding review is just an opportunity or option to obtain grants and does not ensure the securing 
of grants. In other cases, researchers could choose to skip the funding review (Figure 1). For example, they can cover research expenses in another way (e.g., having another grant outside the trinity review system or using crowdfunding); nevertheless, they still want to receive a combined review from scientific and ethical perspectives (Figure 2).

-- Figure 2 around here --

Here, we point out the positive impact of a trinity review. As already mentioned, this system combines the Stage 1 manuscript of RRs with ethics review and funding review to eliminate the redundancy of multiple paperwork for a single study.

Additionally, there are many other potential advantages. First, papers published through the trinity review system will provide a detailed description of ethical considerations. In this system, an ethics review will be conducted, as well as a scientific review, at Stage 1. Consequently, researchers must describe ethical considerations in greater detail in their protocols. This will help readers determine what specific considerations have been made. Furthermore, since scientific and ethics reviews are conducted simultaneously through the same documents, the researchers have no opportunity to arbitrarily modify the original plan regarding ethical considerations without consulting the journal, as per the process of traditional RRs. This will control the problem of changing ethical operations after the completion of the ethics review [12] and hence suppress questionable ethical practices. Second, any protocols using this system will be published regardless of the statistical significance of the results, since the trinity review is based on RRs. This not only prevents file drawer problems [13] and publication bias $[14,15]$, as in the case of traditional RRs, but also brings a benefit for the funders: they can invest in research that is sure to be published. In addition, the RRs prevent questionable research practices or research misconduct, and as a result, increase reproducibility $[3,16]$; the trinity review system also has these benefits. Third, researchers who belong to institutes or departments that do not have an institutional review board and independent researchers who do not belong to any research institution can have an opportunity to review their protocol from an ethical perspective quickly and easily. Fourth, grants given through this system are tied to the protocol, which makes their purpose of funding use clearer and easierthus preventing their abuse - compared to the traditional grant system. Finally, in the trinity review system, peer reviews of scientific and ethical aspects are completely separated. This allows reviewers to concentrate on their assigned aspect (scientific or ethical). Taken together, 
the trinity review system not only eliminates the redundancy of multiple paperwork but also effectively and efficiently contributes toward improving reproducibility and research integrity.

Some difficulties in implementing the trinity review could be raised. One might argue that this will increase the workload for editors. However, the editorial effort will not change substantially, as the editorial process will be almost the same as that for traditional RRs. The key point is that it requires several ethics reviewers, which could lead to a shortage of reviewers and incentives for them. Next, this trinity review is suitable for disciplines and communities that already have some common rules, guidelines, and formats for ethics reviews; otherwise, the journal will need to prepare them, which will incur additional costs. Furthermore, the trinity review system requires ethics reviewers in addition to academic reviewers. Many journals already experience some strain with regard to finding reviewers [17], and the addition of ethics reviews could be a burden to such journals. In the trinity review system, an ethics review will be conducted using a pool of reviewers dedicated to ethics review by applying a pooling system such as the COVID-19 Rapid Reviewers Collaboration [18]. Lastly, if too much funding is budgeted and invested toward one protocol, there may not be enough funding for other protocols. This is not a problem unique to the trinity review system on which RRs are based, as it occurs in existing grant systems. To prevent such overinvestment, it might be helpful to introduce a cap system for each author [19]. The idea is to set an annual budget limit for each individual. Thus, receiving excessive funding for one study may result in a disadvantage for the researcher, encouraging the distribution of funds to other researchers.

\section{Outlook}

In summary, the trinity review is highly likely to benefit the many relevant parties involved in the system (authors, reviewers, readers, and funders). Therefore, it would be worthwhile to introduce the system on a pilot basis to evaluate its effectiveness. Moreover, the trinity review system is beneficial for reproducibility and research integrity because it suppresses questionable practices involving research, ethical considerations, and the use of funds. This method is easier to implement in research areas and specific research topics, where the use of RRs is already popular and well established. In contrast, this system cannot be applied to exploratory and conceptual disciplines (e.g., humanities and theoretical physics) and studies that are not suitable for using RRs (e.g., developing new engineering techniques and optical illusions [20]). Thus, since the trinity review system will probably not become the standard for all research, and because it is not clear whether RRs themselves will continue to be used [21], the system can only play a temporary and tentative role (Figure 2). 
Nevertheless, it would be a good step forward in developing a system that would allow researchers to concentrate on the task at hand, eliminating the waste of effort in their daily work as much as possible. 


\section{Abbreviations \\ PCI: Peer Community In \\ RRs: Registered Reports}

\section{Declarations}

\section{Ethics approval and consent to participate}

Not applicable

\section{Consent for publication}

Not applicable

\section{Availability of data and materials}

Not applicable

\section{Competing interests}

One of the authors is a Managing Board Member of PCI Registered Reports, which is discussed in this article. The authors declare that they have no other competing interests.

\section{Funding}

This research was supported by Japan Society for the Promotion of Science (https://www.jsps.go.jp/english/) KAKENHI Grant Numbers JP16H03079 (Y.Y.), JP17H00875 (Y.Y.), JP18K12015 (Y.Y.), JP19K14482 (K.S.), JP20H04581 (Y.Y.), and JP21H03784 (K.S. and Y.Y.).

\section{Authors' contributions}

Conceptualization: Mori, Takashima, Ueda, Sasaki, Yamada

Funding acquisition: Sasaki, Yamada

Project administration: Yamada

Supervision: Yamada

Visualization: Mori, Takashima, Ueda, Sasaki, Yamada

Writing: Mori, Takashima, Ueda, Sasaki, Yamada

\section{Acknowledgments}


We would like to thank Editage [http://www.editage.com] for editing and reviewing this manuscript for English language. 


\section{References}

1. Chambers, C. (2019). What's next for registered reports? Nature, 573, 187-189. https://doi.org/10.1038/d41586-019-02674-6

2. Nosek, B. A., Ebersole, C. R., DeHaven, A. C., \& Mellor, D. T. (2018). The preregistration revolution. Proceedings of the National Academy of Sciences, 115, 2600-2606. https://doi.org/10.1073/pnas.1708274114

3. Soderberg, C. K., Errington, T. M., Schiavone, S. R., Bottesini, J., Thorn, F. S., Vazire, S., Esterling, K.M., \& Nosek, B. A. (2021). Initial evidence of research quality of registered reports compared with the standard publishing model. Nature Human Behaviour, 5(8), 990-997. https://doi.org/10.1038/s41562-021-01142-4

4. Guraya, S. Y., London, N. J. M., \& Guraya, S. S. (2014). Ethics in medical research. Journal of Microscopy and Ultrastructure, 2(3), 121-126. https://doi.org/10.1016/j.jmau.2014.03.003

5. Page, S. A., \& Nyeboer, J. (2017). Improving the process of research ethics review. Research Integrity and Peer Review, 2(1), 1-7. https://doi.org/10.1186/s41073-017-0038$\underline{7}$

6. Herbert, D. L., Barnett, A. G., Clarke, P., \& Graves, N. (2013). On the time spent preparing grant proposals: An observational study of Australian researchers. BMJ Open, 3(5). https://doi.org/10.1136/bmjopen-2013-002800

7. Munafò, M. R. (2017). Improving the efficiency of grant and journal peer review: Registered reports funding. Nicotine \& Tobacco Research, 19(7), 773-773. https://doi.org/10.1093/ntr/ntx081

8. Chambers, C. D., \& Tzavella, L. (2021). The past, present and future of Registered Reports. Nature Human Behaviour. https://doi.org/10.1038/s41562-021-01193-7

9. Dirnagl, U. (2020). Preregistration of exploratory research: Learning from the golden age of discovery. PLOS Biology, 18(3), e3000690. https://doi.org/10.1371/journal.pbio.3000690

10. Guillemaud, T., Facon, B., \& Bourguet, D. (2019). Peer Community In: A free process for the recommendation of unpublished scientific papers based on peer review. ELPUB 2019 23rd edition of the International Conference on Electronic Publishing, Jun 2019, Marseille, France. https://doi.org/10.4000/proceedings.elpub.2019.23 
11. Pennington, C. R., \& Heim, D. (in press). Reshaping the publication process: Addiction Research and Theory joins Peer Community In Registered Reports. Addiction Research \& Theory. https://doi.org/10.1080/16066359.2021.1931142

12. Rogers, W., Robertson, M. P., Ballantyne, A., Blakely, B., Catsanos, R., Clay-Williams, R., \& Singh, M. F. (2019). Compliance with ethical standards in the reporting of donor sources and ethics review in peer-reviewed publications involving organ transplantation in China: a scoping review. BMJ Open, 9(2), e024473. http://dx.doi.org/10.1136/bmjopen$\underline{2018-024473}$

13. Rosenthal, R. (1979). The file drawer problem and tolerance for null results. Psychological Bulletin, 86(3), 638-641. https://doi.org/10.1037/0033-2909.86.3.638

14. Mahoney, M. J. (1977). Publication prejudices: An experimental study of confirmatory bias in the peer review system. Cognitive Therapy and Research, 1(2), 161-175. https://doi.org/10.1007/BF01173636

15. Sterling, T. D., Rosenbaum, W. L., \& Weinkam, J. J. (1995). Publication decisions revisited: The effect of the outcome of statistical tests on the decision to publish and vice $\begin{array}{llll}\text { versa. } & \text { The American } & \text { Statistician, }\end{array}$ https://doi.org/10.1080/00031305.1995.10476125

16. Protzko, J., Krosnick, J., Nelson, L. D., Nosek, B. A., Axt, J., Berent, M., Buttrick, N., DeBell, M., Ebersole, C. R. Lundmark, E. S., MacInnis, B., O’Donnell, M., Perfecto, H., Pustejovsky, J. E. Roeder, S., Walleczek, J., Schooler, J.W. (2020). High replicability of newly-discovered social-behavioral findings is achievable. PsyArxiv. https://psyarxiv.com/n2a9x/

17. Perrin, W. F. (2008). In Search of Peer Reviewers. Science, 319(5859), 32. https://doi.org/10.1126/science.319.5859.32b

18. Open Access Scholarly Publishing Association. (2021, August 11). Covid-19 publishers Open letter of intent - rapid review. OASPA. Retrieved December 6, 2021, from https://oaspa.org/covid-19-publishers-open-letter-of-intent-rapid-review/

19. Reardon, S. (2017). NIH to limit the amount of grant money a scientist can receive. Nature. https://doi.org/10.1038/nature.2017.21930

20. Kitaoka, A., Kawabe, T., \& Yamada, Y. (2020). Introducing the Journal of Illusion. Journal of Illusion, 1(1). https://doi.org/10.47691/joi.v1.5591

21. Yamada, Y. (2018). How to crack pre-registration: Toward transparent and open science. Frontiers in Psychology, 9:1831.https://doi.org/10.3389/fpsyg.2018.01831 


\section{Figures}

Figure 1.

Overview of the trinity review

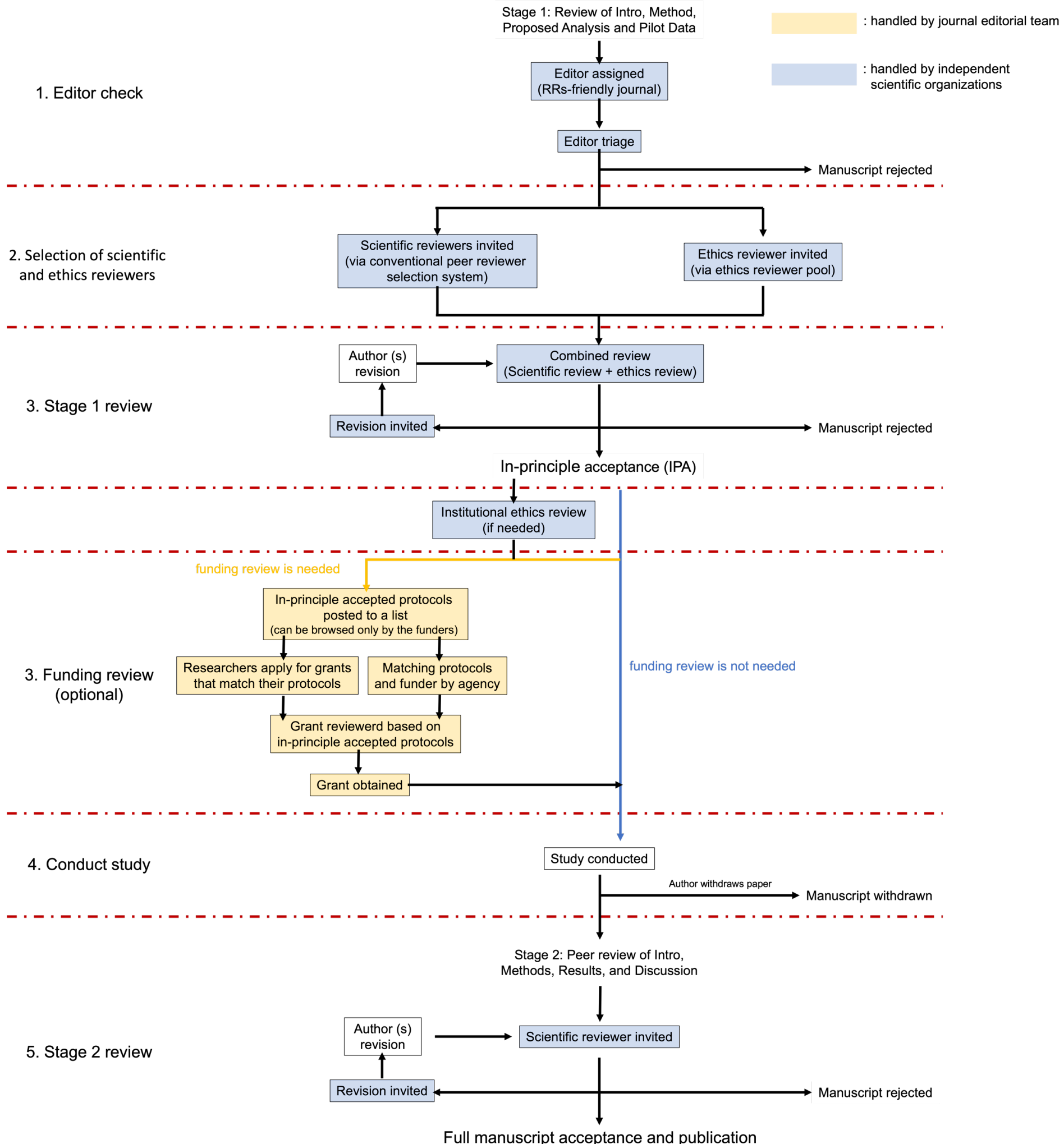

Full manuscript acceptance and publication 
Note. This figure provides an overview of a typical trinity review. The blue rectangles show the processes handled by the journal editorial team, and the yellow rectangles show the processes handled by independent scientific organizations. Each process is described in detail below. 1. Editor check: The editor reads the received manuscript and decides whether to send it for peer review. 2. Selection of scientific and ethics reviewers: The editor assigns suitable scientific and ethics reviewers to the manuscript. The editor selects scientific reviewers in the same way as in the conventional peer review system and selects ethics reviewers from a pool of ethics reviewers. 3. Stage 1 review: The scientific reviewers assess the importance of the research questions of the manuscript, the theoretical validity of the hypothesis derivation process, and whether the experimental method is appropriate for testing the hypothesis. Ethics reviewers assess the protocol for any ethical issues. Scientific and ethics reviews are undertaken concurrently. Once the manuscript has successfully passed both reviews and is accepted in principle, the authors can proceed to the funding review. If ethics reviewers determine that an institutional ethics review is necessary, an ethics review is conducted at the authors' institution. In this case, a funding review is possible only after passing the institutional ethics review. 4. Funding review (matching): If a funding review is required, the editor first adds the manuscript to a list of in-principle accepted protocols that can be browsed only by the funders until the manuscript obtains a grant. There are two ways to obtain funding: the researcher applies for offers that match the protocol, and the funder selects the one of interest from the list of in-principle accepted protocols. If the funding review is not needed, the authors begin conducting the studies immediately. 5. Conduct study: Researchers conduct their studies following the in-principle accepted procedures. 6. Stage 2 review: Following data collection, the authors resubmit a Stage 2 manuscript that includes the Introduction and Methods from the original submission plus the Results and Discussion section. The final article is published after the scientific review for Stage 2 manuscript is completed. 
Figure 2.

The trinity review system as one of the various publishing processes

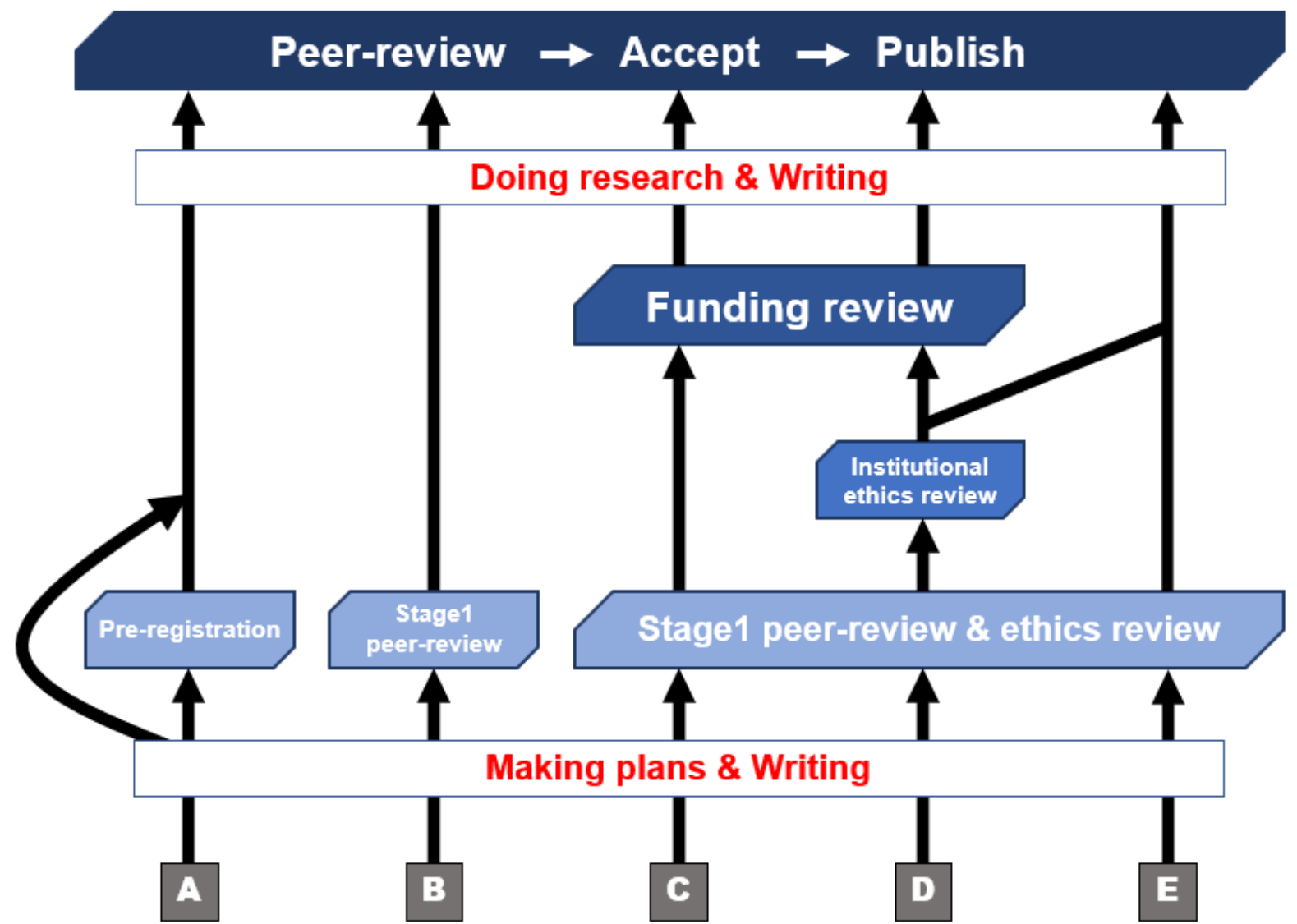

Note. This figure shows the trinity review system as one of the various publication processes and the publication process options available to researchers.

A) Traditional publishing flows. Preregistration by each researcher on a voluntary basis is recommended. B) Flow of the typical and current Registered Reports. At Stage 1, a manuscript that describes methods, plans, hypotheses, and so on in detail is peer-reviewed. After inprinciple acceptance, researchers begin the experiments or investigations. Then, the Stage 2 manuscript, including results and discussion, is peer-reviewed and published. C) The most orthodox flow in the trinity review. An ethics review is conducted at the same time as Stage 1 peer-review for the same manuscript. After in-principle acceptance, a funding review is conducted, and researchers then start experiments or investigations. Finally, the Stage 2 manuscript, including results and discussion, is peer-reviewed and published. More detailed information regarding this flow is shown in Figure 1. D) Manuscripts on the trinity review flow, which have some special necessity, are sent to the institute to which authors belong and are then subjected to a second ethics review. We suppose that the ethics review of the trinity review system is conducted under the code of ethics determined by each journal that approves the trinity review. However, if there is some necessity in terms of ethical considerations that are not covered in the code, the protocol is reviewed along with this flow (e.g., in the medical field, institutional acknowledgement might be necessary if the research uses some invasive methods against humans). Then, researchers start experiments or investigations, and the Stage 2 manuscript will be published after peer-review. E) Trinity review flow without a funding 
review. Researchers who do not need funding for the research but want to utilize the Stage 1 peer-review with ethics review for the same manuscript will use this system. 